\title{
Beam-induced atomic motion in alkali borate glasses
}

\author{
Katharina Holzweber $\odot,{ }^{*}$ Christoph Tietz $\odot$, Tobias Michael Fritz, and Bogdan Sepiol $\odot$ \\ Fakultät für Physik, Universität Wien, Boltzmanngasse 5, 1090 Wien, Austria \\ Michael Leitner® \\ Heinz Maier-Leibnitz Zentrum (MLZ), Technische Universität München, Lichtenbergstraße 1, 85747 Garching, Germany
}

(Received 15 August 2019; revised manuscript received 24 October 2019; published 10 December 2019)

\begin{abstract}
Applying coherent $\mathrm{x}$ rays using the method of atomic-scale x-ray photon correlation spectroscopy results in beam-induced dynamics in a number of oxide glasses. Here these studies are extended to rubidium and cesium borates with varying alkali contents. While no effect due to cumulative beam damage is observed, the observed rate of structural rearrangements shows a linear relation to the instantaneous dose rate. In agreement with the increasing glass transition temperature, the rate of dynamics at a given dose rate decreases with increasing alkali content, while the shape of the decay of correlations becomes progressively stretched. This behavior is explained in terms of faster dynamics of the alkali positions compared to the borate network. Finally, the $q$ dependent behavior of the correlation decay rate implies that the observed dynamics proceeds via small-scale atomic displacements subject to de Gennes narrowing.
\end{abstract}

DOI: 10.1103/PhysRevB.100.214305

\section{INTRODUCTION}

Examining the mechanisms of diffusion in solids on an atomic level is a fundamental issue. Decisive experimental insights have been obtained by the application of classical methods such as quasielastic neutron scattering and Mössbauer spectroscopy [1]. However, the limitation of these methods to comparatively fast diffusivities and specific elements or isotopes has confined such direct investigations to favorable prototypical systems. X-ray photon correlation spectroscopy (XPCS) holds the promise to fill this gap, as it deals with coherent scattering in the time domain. While in the pioneering papers by Sutton et al. [2] and Brauer et al. [3] metallic systems were studied, the majority of later studies concern soft-matter dynamics, with objects in the nanometer range (see, e.g., Refs. [4-9]). The full potential of the method was realized by the first demonstration of atomic-scale XPCS (aXPCS) by Leitner et al. [10]. Since then, it has been applied to several crystalline [11] and amorphous solids [12-14].

For soft condensed matter such as biological samples, the issue of potential beam damage with accumulating dose has always been recognized since XPCS measurements are coupled to powerful synchrotron sources with a highly intense $x$-ray beam. Frequent sample replacement [15], changing the exposure spot on the sample, and introducing low-dose x-ray speckle visibility spectroscopy [16] are ways of mitigating

\footnotetext{
*katharina.holzweber@univie.ac.at
}

Published by the American Physical Society under the terms of the Creative Commons Attribution 4.0 International license. Further distribution of this work must maintain attribution to the author(s) and the published article's title, journal citation, and DOI. this problem. The influence of the x-ray beam on hard condensed matter has been, however, more or less neglected and rarely mentioned so far [17].

A recent remarkable observation during aXPCS measurements on silicate and germanate glasses was reported by Ruta et al. [18]: In these systems, there is a clear dependence of the atomic motion on the incident $\mathrm{x}$-ray flux, which implies that the observed dynamics is driven by the absorbed x-ray intensity. Still, no significant structural modification of the sample is evident. This is in stark contrast to studies on crystalline alloys or metallic glasses, where such an effect is not seen $[17,18]$.

The structure and dynamics of alkali borate glasses, which are prototypical solid-state ionic conductors and thus of great technological interest, are still topics of current research (e.g., [19]). While the accessibility of beam-driven dynamics in the borate end-member system was established in a recent publication [20], it is not yet known how the addition of alkali oxides, which leads to well-established anomalies in a number of static and dynamical properties, collectively known as the borate anomaly [21], affects the susceptibility to a beam-driven acceleration of dynamics, much less how the rearrangements actually proceed on a microscopic scale.

Here we present a detailed study of beam-induced dynamics by aXPCS in rubidium and cesium borate glasses of different alkali contents supported by measurements of the glass transition temperature. Varying the incident intensity by placing attenuators in the beam, we verify the expected linear relation of incident beam intensity with the resulting dynamics and identify the absorbed dose rate as the fundamental deciding quantity. Comparing timescales and shape parameters of the intensity fluctuation correlation functions for isoelectronic $\mathrm{Rb}$ - and Cs-based systems, we can distinguish the dynamics of the alkali sites from those of the borate network, while 
studying the correlation decay rate as a function of $q$ allows us to conclude that the structural rearrangements are dominated by small-scale atomic displacements as opposed to discrete jumps on length scales comparable to atomic distances.

\section{INTENSITY AUTOCORRELATION: THEORY}

Introductions to the theory and practice of XPCS can be found in Refs. [22-24]. The basic idea is to quantify the timescale of temporal fluctuations of the electron density on the atomic scale as probed by coherent $\mathrm{x}$-ray scattering via the intensity autocorrelation function

$$
g^{(2)}(\vec{q}, \Delta t)=\frac{\langle I(\vec{q}, t) I(\vec{q}, t+\Delta t)\rangle}{\langle I(\vec{q}, t)\rangle^{2}} .
$$

Here $I(\vec{q}, t)$ is the observed intensity at scattering vector $\vec{q}$ and at time $t$, and the brackets $\langle\cdots\rangle$ denote the ensemble average, which experimentally is realized as an average over absolute time $t$ as well as over the corresponding pixels on the detector. This quantity can be related to the normalized intermediate scattering function $F(\vec{q}, t)$,

$$
g^{(2)}(\vec{q}, \Delta t)=1+A(\vec{q})[F(\vec{q}, t)]^{2},
$$

where $A(\vec{q})$ is the coherence factor with values between 0 and 1 quantifying the degree of coherence [25]. Via a Fourier transformation in space $F(\vec{q}, t)$ is related to the Van Hove pair correlation function $G(\vec{r}, t)$ that enables information about the dynamics of particles.

Dynamical rearrangements in the sample lead to fluctuating scattered intensities and thus to a decay of the autocorrelation function. Empirically, the expression

$$
F(\vec{q}, \Delta t)=\exp (-\Gamma(\vec{q}) \Delta t)^{\beta},
$$

known as Kohlrausch-Williams-Watts (KWW) function, has been found to satisfactorily describe diverse systems [26]. A system of noninteracting identical random walkers or a concentrated noninteracting lattice gas with site exclusion would give an ideal exponential decay with shape parameter $\beta=1$, while the different local environments in a disordered glass let us expect dynamics on varying timescales, leading to a stretched exponential decay with $\beta<1$. Still, the primary fit parameter is the decay constant $\Gamma(\vec{q})$, whose variation with $\vec{q}$ encodes the spatial scale of structural rearrangements. From now on $\vec{q}$ will be replaced by $q$ due to the isotropy of glasses.

\section{EXPERIMENTAL APPROACH}

\section{A. Sample preparation}

Rubidium and cesium borate glasses with different compositions $\left(\mathrm{A}_{2} \mathrm{O}\right)_{x}\left(\mathrm{~B}_{2} \mathrm{O}_{3}\right)_{1-x}$ have been prepared with molar fractions of $x=0,0.02,0.10,0.15,0.20$, and 0.30 . The chemically pure materials were mixed and melted in alumina crucibles in an electrically heated muffle furnace at a temperature of $1273 \mathrm{~K}$ for about $3 \mathrm{~h}$. To avoid inhomogeneities in the glass the melt was stirred several times and subsequently poured into a cylindrical brass mold. After the preparation the glasses were heat treated at a temperature of about $20 \mathrm{~K}$ below $T_{\mathrm{g}}$ for $3 \mathrm{~h}$ and then slowly cooled down to room temperature with cooling rates on the order of $1 \mathrm{~K} / \mathrm{min}$ in order to reduce tension in the vitreous materials. As alkali
TABLE I. Density $\rho$, position of the first diffraction peak $q_{\max }$, and absorption length $1 / \mu$ at $13 \mathrm{keV}$ of $\left(\mathrm{Rb}_{2} \mathrm{O}\right)_{x}\left(\mathrm{~B}_{2} \mathrm{O}_{3}\right)_{(1-x)}$.

\begin{tabular}{lccccc}
\hline \hline$x$ & 0.02 & 0.10 & 0.15 & 0.20 & 0.30 \\
\hline$\rho\left(\mathrm{g} / \mathrm{cm}^{3}\right)$ & 1.98 & 2.22 & 2.39 & 2.50 & 2.84 \\
$q_{\max }\left(\AA^{-1}\right)$ & 1.62 & 1.70 & 1.81 & 1.82 & 1.86 \\
$1 / \mu(\mu \mathrm{m})$ & 1646 & 642 & 462 & 369 & 255 \\
\hline \hline
\end{tabular}

borates are hygroscopic, they were kept in vacuum during the measurement and stored in a dry atmosphere at all other times.

The densities were measured with the method of Archimedes using decahydronaphthalene as the medium and are given in Tables I and II. The glass transition temperatures were determined by a Netzsch DSC 204 device employing a heating rate of $20 \mathrm{~K} / \mathrm{min}$. To erase any effects of the previous thermal history, we first cycled through the glass transition and determined the inflection points only in the second heating cycle. The corresponding values are given in Fig. 1.

The actual specimens for the aXPCS measurements were cut by a low-speed diamond saw, ground, and polished to slabs of about $0.2 \mathrm{~mm}$ thickness. Then, using a dimpling grinder with a wheel radius of $7.5 \mathrm{~mm}$, a hole in the shape of a spherical segment was excavated in order to allow choosing the sample thickness during the experiment by translating the sample table laterally. With beam sizes of a few micrometers as discussed below, the illuminated part of the sample can be considered plane parallel. Note that for these systems, the penetration lengths at $13 \mathrm{keV}$ as used in the aXPCS measurements shown in Tables I and II are much higher than the longitudinal coherence length of the beam. Thus, in contrast to the situation typical of small-angle XPCS [27], the optimal sample thickness results from the requirement of achieving a reasonable contrast for coherent $\mathrm{x}$-ray measurements rather than from maximizing the scattered radiation. We typically used thicknesses of about $30 \mu \mathrm{m}$.

\section{B. aXPCS measurements}

The aXPCS measurements were conducted at beamline P10 of the synchrotron PETRA III using a coherent setup with $13 \mathrm{keV}$ photons and an estimated flux of $\Phi_{0}=10^{11}$ photons $/ \mathrm{s}$. To study the dynamics under different photon fluxes, variable numbers of attenuator plates made of $\mathrm{Ag}$ or $\mathrm{Si}$ were introduced into the beam, with thicknesses per plate of 12.5 and $25 \mu \mathrm{m}$, respectively, corresponding to transmission coefficients at $13 \mathrm{keV}$ of $T_{\mathrm{Ag}}=0.483$ and $T_{\mathrm{Si}}=0.917$ per plate. Using a system of refractive $\mathrm{x}$-ray lenses, a beam spot with FWHM of $2.5 \times 2 \mu \mathrm{m}^{2}(\mathrm{~h} \times \mathrm{v})$

TABLE II. Density $\rho$, position of the first diffraction peak $q_{\max }$, and absorption length $1 / \mu$ at $13 \mathrm{keV}$ of pure $\mathrm{B}_{2} \mathrm{O}_{3}$ and $\left(\mathrm{Cs}_{2} \mathrm{O}\right)_{x}\left(\mathrm{~B}_{2} \mathrm{O}_{3}\right)_{(1-x)}$.

\begin{tabular}{lccccc}
\hline \hline$x$ & 0.00 & 0.02 & 0.10 & 0.15 & 0.20 \\
\hline$\rho\left(\mathrm{g} / \mathrm{cm}^{3}\right)$ & 1.81 & 1.92 & 2.36 & 2.54 & 2.75 \\
$q_{\max }\left(\AA^{-1}\right)$ & 1.59 & 1.70 & 1.75 & 1.76 & 1.77 \\
$1 / \mu(\mu \mathrm{m})$ & 2884 & 634 & 154 & 108 & 84 \\
\hline \hline
\end{tabular}




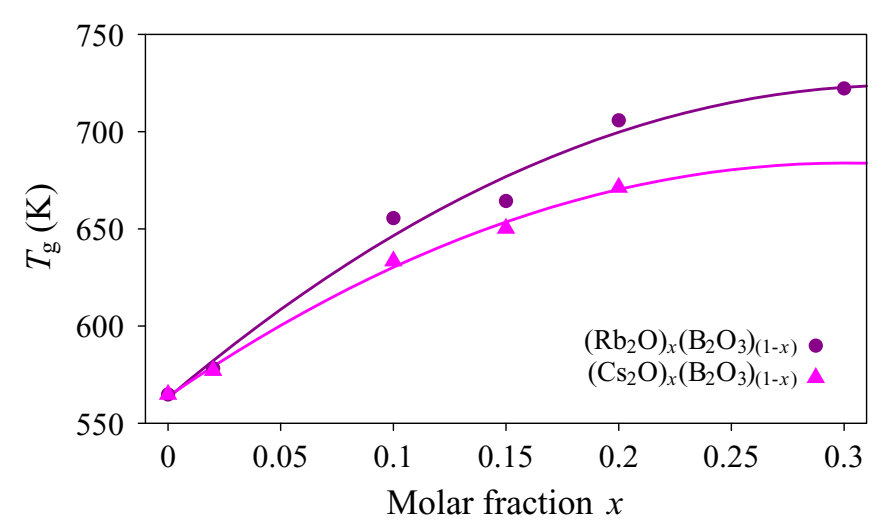

FIG. 1. Glass transition temperatures $T_{g}$ for different concentrations of $\left(\mathrm{Rb}_{2} \mathrm{O}\right)_{x}\left(\mathrm{~B}_{2} \mathrm{O}_{3}\right)_{(1-x)}$ and $\left(\mathrm{Cs}_{2} \mathrm{O}\right)_{x}\left(\mathrm{~B}_{2} \mathrm{O}_{3}\right)_{(1-x)}$.

was achieved. The sample was mounted in a custom-built chamber in transmission geometry at a vacuum of $\approx 10^{-6}$ mbar. All experiments were performed at room temperature. Scattered photons were recorded by the Eiger X 4M detector with a pixel size of $75 \times 75 \mu \mathrm{m}^{2}$, a continuous readout time, and a negligibly low dead time. To suppress fluorescence an appropriate energy threshold was chosen. With a sampledetector distance of about $181 \mathrm{~cm}$ and a usable detector width of about $14.5 \mathrm{~cm}$ (the actual side length of this detector is higher, but approximately $1 / 3$ of the area was shadowed by the flight tube) an angle of about $4.5^{\circ}$ was covered for a given detector setting.

A single measurement run typically consisted of 1000 frames and accumulation times of 0.1 to $1 \mathrm{~s}$. To control temporal instabilities or cumulative effects, we frequently and nonsystematically switched between, respectively, the different incident fluxes $\Phi_{\text {in }}$ and scattering vectors $2 \theta$ and generally measured each setting repeatedly.

To increase the angular resolution, we divided the detector area into $N$ slices and evaluated our data with respect to the total diffracted intensity by summing over all pixels and frames (with $N=12$ ), and we computed the intensity autocorrelation function for each run by averaging over the pixels with $N=2$ slices. We also computed two-time correlation functions $C\left(t_{1}, t_{2}\right)$ [26] for each measurement to check the stability of the sample and the mechanical stability of the setup. We report the diffracted intensity as a function of the accumulated dose. On the other hand, we observed no effect of the accumulated dose on the dynamical properties, which allowed us to average the autocorrelation functions for given $\Phi_{\text {in }}$ and $2 \theta$. Decay constants $\Gamma$ and shape parameters $\beta$ as functions of dose rate and wave vector $q$ were obtained by fitting Eq. (3).

\section{RESULTS AND DISCUSSION}

\section{A. Beam-induced effect on structural properties}

To examine the beam-induced effect on the structure, we evaluated the intensity at the diffraction peak as a function of elapsed time. As Fig. 2(a) shows, there are no discernible continuous or abrupt changes in intensity on short timescales while collecting a series of frames. This is valid for the first measurement [in Fig. 2(a) shown for $\left(\mathrm{Rb}_{2} \mathrm{O}\right)_{15}\left(\mathrm{~B}_{2} \mathrm{O}_{3}\right)_{85}$ with an incoming flux of $0.48 \Phi_{0}$ ] as well as for all further measurements with different incoming fluxes, where we retained the sample position.
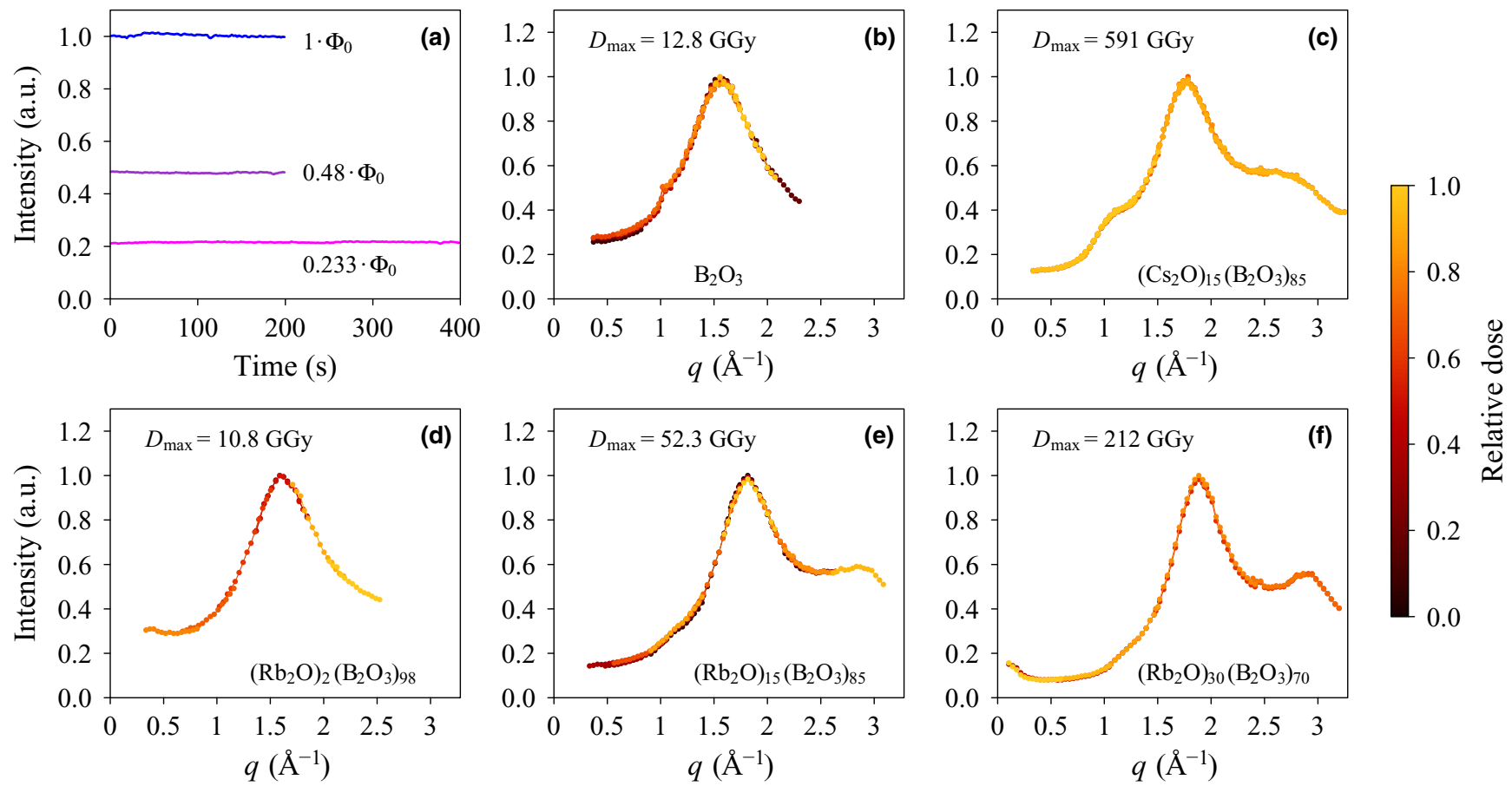

FIG. 2. Quantification of beam damage under irradiation: (a) shows the time evolution of the intensity $I(t)$ for $\left(\mathrm{Rb}_{2} \mathrm{O}\right)_{15}\left(\mathrm{~B}_{2} \mathrm{O}_{3}\right)_{85}$ measured under different incoming $\Phi_{\mathrm{in}}$ at $q_{\max }$. (b)-(f) show the evolution of $I(q)$ with accumulated dose for the various systems, with the dose relative to its final value encoded in the color of the curves. 
For a quantitative discussion of irradiation effects, it is, of course, the dose rate rather than the incident flux that is relevant. Modeling the beam profile as an anisotropic Gaussian function with $d_{1}$ and $d_{2}$ as the principal FWHMs gives a radiative flux density of $2 \ln (2) \Phi_{0} / \pi d_{1} d_{2}$ averaged over the profile of the beam, which evaluates to about $8.825 \times 10^{21}$ photons per second and square meter for the above-mentioned parameters at full beam. Multiplying by the respective absorption cross sections, this corresponds to dose rates $S$ of a few $\mathrm{eV}$ per atom and second, or some $10 \mathrm{MGy} / \mathrm{s}$.

Figures 2(b)-2(f) report our results on the dose-dependent diffraction curves, spliced together from the measurements at different detector positions for constant incident flux. Indeed, particularly for $\mathrm{B}_{2} \mathrm{O}_{3}$ and $\left(\mathrm{Rb}_{2} \mathrm{O}\right)_{15}\left(\mathrm{~B}_{2} \mathrm{O}_{3}\right)_{85}$, which are the systems with data also from very early stages, there are indications of a slight flattening of the diffraction curves effected by a transfer of intensity from the peak specifically to low- $q$ regions, pointing to beam damage. However, the corresponding structural modification as quantified by the atomic pair distribution functions is very small, probably on the same level as the degree of reproducibility when preparing such samples of a given composition and annealing protocol in the first place. Thus, our conclusion of small to negligible structural effects is in agreement with the work of Pintori et al. [20].

\section{B. Beam-induced effect on dynamical properties}

Alkali borate glasses are described by the borate anomaly [21]: The charge diffusion [28] and the glass transition temperature $T_{g}$ (Fig. 1) increase with increasing alkali content and reach a shallow maximum at about $x \approx 0.3$ of the alkali oxide mole fraction. From a microscopic point of view this is predominately achieved by the modification of the borate network from $\mathrm{BO}_{3}$ to $\mathrm{BO}_{4}$ structural units [19,29]. Specifically, in pure $\mathrm{B}_{2} \mathrm{O}_{3}$ the boron atoms are in symmetric threefold oxygen coordination. The incorporation of $\mathrm{A}_{2} \mathrm{O}$ units supplies additional oxygen atoms, causing a partial increase to fourfold coordination. Four instead of only three bonds of the tetrahedrons could be a simple reason for the increasing rigidity of the system as evidenced by the rise of $T_{\mathrm{g}}$. The positively charged ions preferentially reside next to the vicinity of negatively charged $\mathrm{BO}_{4}$ tetrahedrons and in this constellation exhibit a very high mobility within the borate network. Beyond an alkali oxide concentration of $x=0.3$ the ions are more strongly bound to the emerging nonbridging oxygens of $\mathrm{BO}_{3}$ entities that gradually replace the tetrahedrons. Hence, the ionic mobility and $T_{g}$ reverse their trend.

Based on low charge diffusion values, e.g., $\approx 10^{-29}\left(\mathrm{~m}^{2} / \mathrm{s}\right)$ for $\left(\mathrm{Rb}_{2} \mathrm{O}\right)_{15}\left(\mathrm{~B}_{2} \mathrm{O}_{3}\right)_{85}$ at room temperature [28], no thermal structural rearrangements visible by aXPCS can be expected for most of our systems. However, Pintori et al. [20] demonstrated that the $\mathrm{x}$-ray beam used to probe dynamics in aXPCS does give rise to measurable dynamics in pure borate glass and that the effect is more pronounced for stronger irradiation. Figure 3(a) shows that this effect can, indeed, be reproduced in $\left(\mathrm{Rb}_{2} \mathrm{O}\right)_{30}\left(\mathrm{~B}_{2} \mathrm{O}_{3}\right)_{70}$ : The decay of the normalized intensity autocorrelation function $g_{2}(q, t)$ measured at the first maximum peak of $I\left(q_{\max }\right)$ shifts towards longer times when attenuating the incident flux, revealing a slowing of dynamics
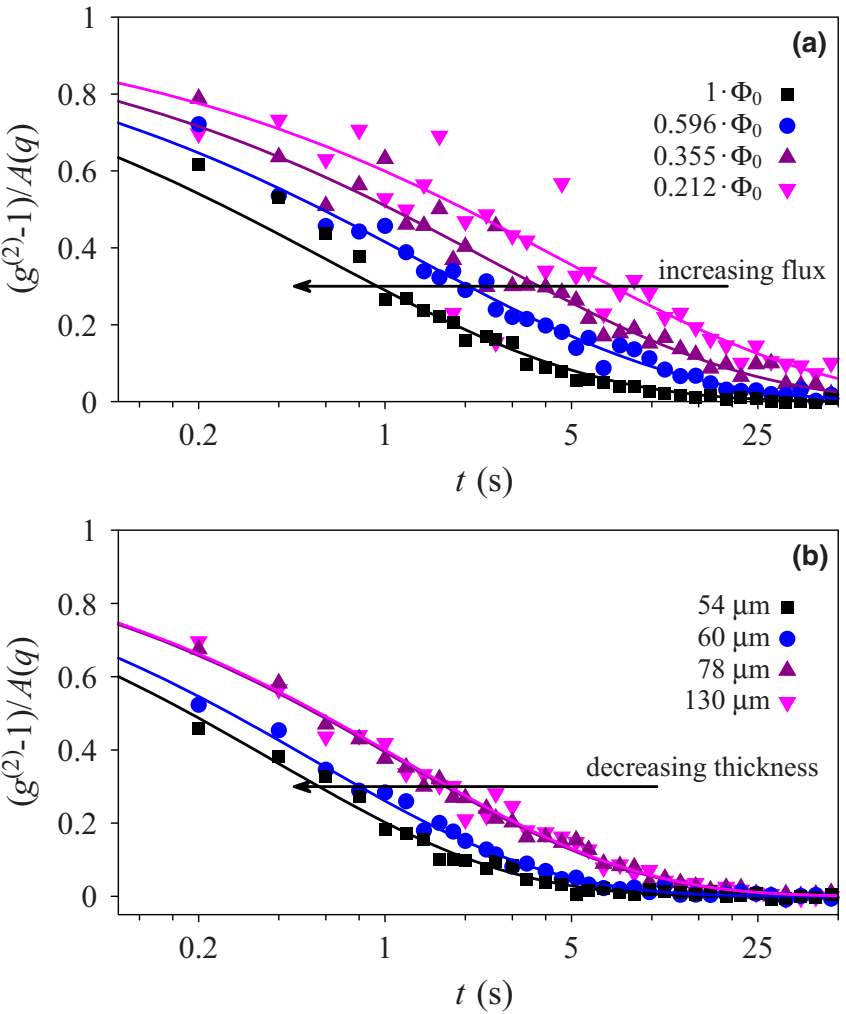

FIG. 3. Beam-driven dynamics in $\left(\mathrm{Rb}_{2} \mathrm{O}\right)_{30}\left(\mathrm{~B}_{2} \mathrm{O}_{3}\right)_{70}$ : The normalized intensity autocorrelation functions measured at $q_{\max }$ shift towards larger times for (a) decreasing incident flux $\Phi_{\text {in }}=T_{x} \Phi_{0}$ with the transmission coefficient $T_{x}$ as well as (b) increasing thickness. Lines are fits with a KWW function with a common shape factor $\beta$ in (a) and thickness-dependent $\beta$ in (b).

with decreasing dose rate as expected for irradiation-driven dynamics.

Performing series of measurements on various target positions with different thicknesses also shows a change in the decay rate of $g^{(2)}(q, t)$. Specifically, Fig. 3(b) shows that the decay rate decreases with increasing thickness. This becomes obvious considering the Beer-Lambert law $\Phi(x)=\Phi_{\text {in }} e^{-\mu x}$, implying that also the rate of absorbed photons declines exponentially when traversing the sample.

A detailed study of the relation between dose rate and induced dynamics in the exemplary system $\left(\mathrm{Rb}_{2} \mathrm{O}\right)_{30}\left(\mathrm{~B}_{2} \mathrm{O}_{3}\right)_{70}$ is reported in Fig. 4. Here as well as in the following discussion of the dose-rate-dependent dynamics in the other systems, for a given system all autocorrelation functions have been fitted with a common coherence factor $A(q)$ and shape parameter $\beta$ and independent decay constants $\Gamma$. Apart from the qualitatively expected monotonic acceleration of dynamics with dose rate, two points may be noted: First, in the regime of comparatively low dose rates, the relation is perfectly linear. Specifically, it is very suggestive that the extrapolation to zero dose rate would, indeed, correspond to practically frozen dynamics. Thus, no thermal dynamics accessible by aXPCS are expected at room temperature in this system. On the other hand, at higher dose rates a significant upward deviation from the linear relationship is obvious in $\left(\mathrm{Rb}_{2} \mathrm{O}\right)_{30}\left(\mathrm{~B}_{2} \mathrm{O}_{3}\right)_{70}$. We will discuss this point in more detail below. 


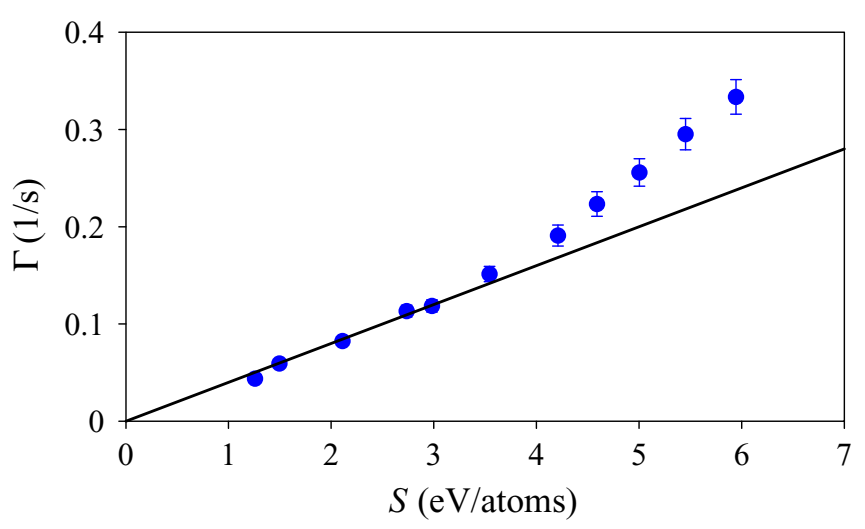

FIG. 4. $\Gamma$ as a function of average dose rate $S$ in $\left(\mathrm{Rb}_{2} \mathrm{O}\right)_{30}\left(\mathrm{~B}_{2} \mathrm{O}_{3}\right)_{70}$ measured at $q_{\max }$. The blue solid circles indicate a measurement series of different combinations of absorbers, while the line corresponds to a strictly linear proportionality.

In contrast to the clear instantaneous effect of dose rate on dynamics, we do not find a significant modification of the dynamics with accumulated dose. This shows that the slight flattening of the diffraction curves discussed above is, indeed, insignificant from the point of view of dynamics and agrees with the findings of Ruta et al. [18] that the structural dynamics of $\mathrm{SiO}_{2}$ follow the dose rate instantaneously and reversibly.

The linear relationship between dose rate and dynamics is reproduced very satisfactorily also in the other systems, as illustrated in Fig. 5. This figure gives the impression that at a given dose rate the dynamics become slower with increasing alkali content, while the $\mathrm{Rb}$ and $\mathrm{Cs}$ systems for the same alkali oxide content $x$, which a priori would be expected to be chemically quite similar, show noticeable differences, with the Cs-based system in general being faster.

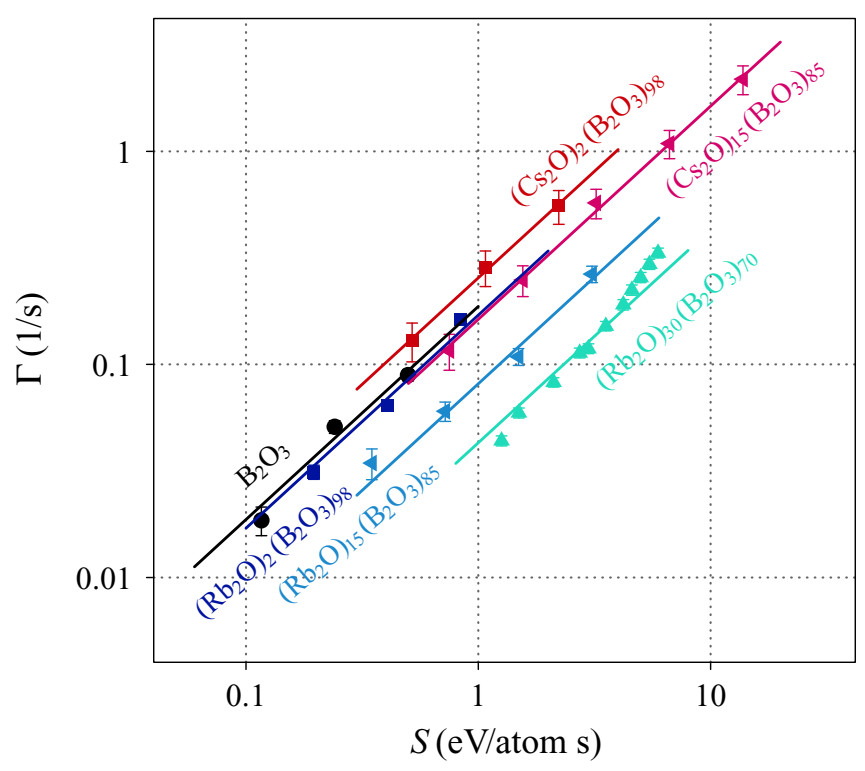

FIG. 5. Decay constant $\Gamma$ at the respective structure peaks for the different systems as a function of dose rate together with fits assuming a linear proportionality.
TABLE III. Dynamical properties $\Gamma$ and $\beta$ at structural peak $q_{\max }$ and evaluated for a dose rate $S=1 \mathrm{eV}$ per atom and second for the different systems, together with the partial structure factor for the alkali atoms $S_{\mathrm{AA}}$, corresponding to their relative contribution to the scattered intensity.

\begin{tabular}{llcc}
\hline \hline System & $\Gamma(1 / \mathrm{s})$ & $\beta$ & $S_{\text {AA }}$ \\
\hline $\mathrm{B}_{2} \mathrm{O}_{3}$ & $0.187(8)$ & $0.790(63)$ & 0 \\
$\left(\mathrm{Rb}_{2} \mathrm{O}\right)_{2}\left(\mathrm{~B}_{2} \mathrm{O}_{3}\right)_{98}$ & $0.170(6)$ & $0.813(56)$ & 0.205 \\
$\left(\mathrm{Cs}_{2} \mathrm{O}\right)_{2}\left(\mathrm{~B}_{2} \mathrm{O}_{3}\right)_{98}$ & $0.255(28)$ & $0.525(65)$ & 0.370 \\
$\left(\mathrm{Rb}_{2} \mathrm{O}\right)_{15}\left(\mathrm{~B}_{2} \mathrm{O}_{3}\right)_{85}$ & $0.081(4)$ & $0.545(39)$ & 0.692 \\
$\left(\mathrm{Cs}_{2} \mathrm{O}\right)_{15}\left(\mathrm{~B}_{2} \mathrm{O}_{3}\right)_{85}$ & $0.163(12)$ & $0.414(24)$ & 0.832 \\
$\left(\mathrm{Rb}_{2} \mathrm{O}\right)_{30}\left(\mathrm{~B}_{2} \mathrm{O}_{3}\right)_{70}$ & $0.0429(7)$ & $0.436(12)$ & 0.838 \\
\hline \hline
\end{tabular}

However, it has to be noted that the probed dynamics in aXPCS is to be understood as an average over the dynamics of the constituent elements, which are likely to be different. Further, due to the much larger scattering cross section of Cs compared to $\mathrm{Rb}$, at a given alkali oxide content $x$ the alkali contribution will be larger in the Cs-based system than in the $\mathrm{Rb}$-based system. The corresponding values calculated under an assumed absence of short-range order are given in Table III, together with the decay rates $\Gamma$ evaluated in each system for a dose rate $S$ of $1 \mathrm{eV}$ per atom and second and the shape parameters $\beta$. The following two hypotheses are now sufficient for explaining the observed behavior as discussed in the following paragraph:

(i) Consistent with the behavior of the glass transition temperature, the structural rearrangements become slower for increasing alkali oxide content $x$, where the introduction of either $\mathrm{Rb}_{2} \mathrm{O}$ or $\mathrm{Cs}_{2} \mathrm{O}$ into the matrix is, in the first approximation, equally effective.

(ii) In any system, the dynamics of rearrangements of the alkali sites is faster than that of the borate network.

Starting with $\mathrm{B}_{2} \mathrm{O}_{3}$, we see that the dynamics of the borate network is described by a shape parameter $\beta$ close to 1 , as expected for a strong glass former [30] and as also found previously [20]. Introducing a small amount of alkali oxide $(x=0.02)$ does not have a large effect (compare Fig. 1), so that the values for $\left(\mathrm{Rb}_{2} \mathrm{O}\right)_{2}\left(\mathrm{~B}_{2} \mathrm{O}_{3}\right)_{98}$ stay essentially the same. On the other hand, when introducing the same amount of $\mathrm{Cs}_{2} \mathrm{O}$, the weight of the alkali sites in the scattered intensity becomes noticeable, leading first to a faster decay rate (as it was assumed that the alkali sites have faster dynamics than the borate network), and further show up in a decrease of the shape parameter $\beta$ that reflects the scattered intensity being made up of contributions with differing dynamics. At an alkali oxide content of $x=0.15$, the overall slowing of dynamics becomes apparent. Now also the $\mathrm{Rb}_{2} \mathrm{O}$-based system has a sizable contribution from the alkali sites to the scattering and thus a decreased $\beta$, while in the $\mathrm{Cs}_{2} \mathrm{O}$-based system the alkali scattering dominates strongly, leading to faster apparent dynamics with a small shape parameter $\beta$ that reflects the pertinent autocorrelation function being composed of a fast component with a large amplitude on top of a slow component with a small amplitude. At $x=0.30$, the overall deceleration via the stabilization of the glass network by the alkali ions has progressed even further. Note that this slowing down 

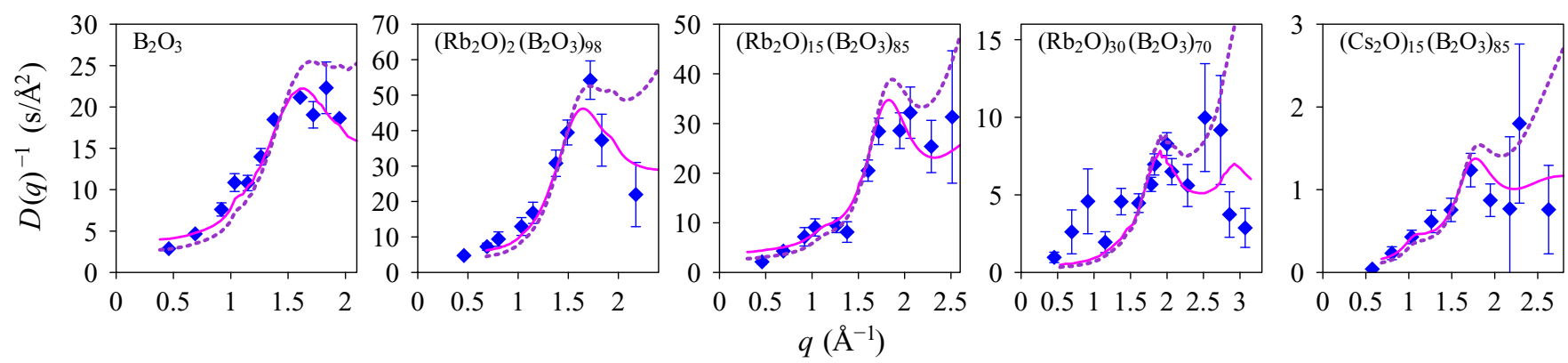

FIG. 6. Inverse $q$-dependent diffusion constant $D(q)^{-1}$. Apart from $\left(\mathrm{Rb}_{2} \mathrm{O}\right)_{2}\left(\mathrm{~B}_{2} \mathrm{O}_{3}\right)_{98}$ and $\left(\mathrm{Rb}_{2} \mathrm{O}\right)_{15}\left(\mathrm{~B}_{2} \mathrm{O}_{3}\right)_{85}$, which were measured with a beam attenuated by a factor of 0.483 , all were measured at full beam. The data are compared to the corresponding IBM models (solid pink lines) and the Chudley-Elliott prediction with jump length $l=\pi / q_{\max }$ taking de Gennes narrowing into account, as discussed in the text (dashed purple lines).

of structural rearrangements with increased alkali content is quite unrelated to ionic diffusion, as the latter accelerates in the corresponding situation [28].

\section{Spatial aspects of dynamics}

We now turn to an explicit microscopic modeling of the structural rearrangements that give rise to the temporal fluctuations in the coherently scattered intensity as studied by aXPCS. Such information is accessible by studying, different from the previous section, how the decay rate $\Gamma(q)$ varies also away from the structural peak at $q_{\text {max }}$.

The simplest model for diffusive dynamics is to assume that the atoms independently perform a random walk in the Brownian sense, composed of microscopic steps below the resolution of observation and thus assumed to be infinitesimal. In this case, the decay rate has the particularly simple form of

$$
\Gamma_{\mathrm{BM}}(q)=q^{2} D_{\mathrm{BM}},
$$

where $D_{\mathrm{BM}}$ is the diffusion constant corresponding to the Brownian motion. Conversely, this relation suggests reducing our experimentally observed decay constants to $q$-dependent diffusion "constants" [31],

$$
D(q)=\Gamma(q) / q^{2},
$$

which would be independent of $q$ under the assumption of the atoms performing independent Brownian motion. Measurement series of $\Gamma(q)$ for the small and intermediate $q$ range in all systems considered here are reported in this way in Fig. 6.

Obviously, the $q$-dependent diffusion constants are far from being constant, which rules out the naive assumption of the atoms in our alkali borate glasses performing independent Brownian motion. In contrast, we observe over all systems a clear increase of $D(q)^{-1}$ towards a maximum at about $q=1.8 \AA^{-1}$. This is reminiscent of the structure factor $S(q)$, and indeed, it was initially proposed for quasielastic neutron scattering on liquids [32] and later verified for aXPCS in hard condensed matter [33] that the simplest modification to incorporate preferential atomic arrangements reflected in a nonconstant structure factor $S(q)$ into a given model of independent microscopic motion is to divide the decay constant $\Gamma(q)$ by the structure factor $S(q)$. We term the resulting model

$$
D_{\mathrm{IBM}}(q)=\frac{D_{\mathrm{BM}}}{S(q)}
$$

an interacting Brownian motion (IBM) model, which obviously fits our data very satisfactorily. The effect of a slowing of dynamics where the structure factor is high is known as de Gennes narrowing.

To demonstrate the significance of the fit, we test also a contrary scenario of jumps of fixed length $l$ in random directions. In the resulting Chudley-Elliott model [34] the decay constant is given by

$$
\Gamma_{\mathrm{CE}}(q)=\Gamma_{0}[1-\sin (q l) / q l],
$$

with a jump frequency of $\Gamma_{0}$. Note that via the Einstein relation $D=\Gamma_{0} l^{2} / 6$ for small $q$ the Chudley-Elliott behavior reduces to Eq. (4) describing Brownian motion, while $\Gamma_{\mathrm{CE}}(q)$ converges to $\Gamma_{0}$ for $q \gtrsim 1 / l$ and thus stands in contrast to the perfect parabolic increase of $\Gamma_{\mathrm{BM}}(q)$. In particular, in the scenario proposed by Pintori et al. for pure borate glass [20], they assume a total decorrelation of the scattering phase after an atomic jump when measuring at $q_{\max }$, which implies that the atoms perform jumps of a finite length of at least $l=\pi / q_{\max }$. Taking de Gennes narrowing into account and plotting the corresponding model in Fig. 6 shows that the predicted leveling off of $\Gamma(q)$, corresponding to an increase of $D(q)^{-1}$, is not in agreement with the data. This rules out their microscopic picture where the effect of an absorbed photon is restricted to a comparatively small number of atoms, but each of these atoms is displaced over a distance on the order of $\pi / q_{\max }$, as indeed has been found in lead silicate glasses [12]. In contrast, our $q$-resolved data show that the microscopic displacements can be regarded with good accuracy as infinitesimal on any probed scale. That is, in their majority they have to be much smaller than the typical atomic distances.

\section{MICROSCOPIC PICTURE OF BEAM-INDUCED DYNAMICS}

In our view, the most plausible model in accordance with the available data is the following: In the majority of cases, the primary event of photoabsorption happens with the emission of an electron from one of the lowest accessible core levels in the system. The resulting deep hole is deexcited by emission of a fluorescence photon, in which case the process of absorption is started anew at somewhat lower energy, or by an Auger electron. In either case, the x-ray photon energy is initially transferred into the electron system in the form of 
a few electrons in the $\mathrm{keV}$ range. These mobile high-energy electrons will then inelastically interact with other electrons, specifically breaking covalent bonds and leading to structural rearrangements.

In the initial report on the observation of x-ray beaminduced dynamics, Ruta et al. called this process radiolysis [18]. While this term captures the essence of the athermal breaking of bonds, we feel it necessary to point out two main differences from classical cases of radiolysis such as the decomposition of water by alpha radiation: First, here it is not the primary ionizing radiation (in our case the $\mathrm{x}$-ray photons) that breaks up the majority of the bonds, but rather the emitted Auger and photoelectrons.

Further, the absence of large-scale permanent beam damage as discussed in Sec. IV A shows that the breaking of the bonds is only transient: The ejection of electrons from a covalent bond due to the inelastic collisions with the $\mathrm{keV}$ range photo- and Auger electrons leads to a local electron deficiency (and at the same time an electron excess at the sites where the ejected electrons come to rest again). The borate and alkali borate glasses are insulators with respect to electronic current. Thus, these localized charges have a finite lifetime during which the electronic structure will decay to its new local ground state subject to the non-neutral charge. As a consequence, on timescales characteristic of phononic dynamics (picoseconds) the atoms will then relax in this modified potential as resulting in the Born-Oppenheimer picture to a new configuration, probably characterized by increased or decreased coordinations determined by the localized charge state. Still, even in insulators electrons have a finite mobility, which is further aided by disturbances in the electronic structure due to the continuing absorption of photons, so that at even later times the locally neutral charge state with the most advantageous atomic coordinations will be restored. However, the inherent geometrical frustration in these systems, which is the reason for the glassy state being stable at all, implies that there are a number of local configurations more or less equivalent in energy the system can relax into. What leads to structural dynamics on the timescale of seconds as visible in aXPCS are those events where the final state after restoration of local charge neutrality is different from the initial state. As the rearrangements proceed via athermal relaxations towards new local ground states due to modified ionic potentials rather than thermal transitions over energy barriers, the large mass differences between chemically equivalent $\mathrm{Rb}$ - and Cs-based borate systems will not affect the timescale of dynamics, different from the classical isotope effect of diffusion [35].

According to our data (see Fig. 5) and in perfect agreement with Pintori et al. [20], a timescale of intensity fluctuations at the structural maximum of about $1 \mathrm{~s}$ corresponds to dose rates on the order of $10 \mathrm{eV} / \mathrm{s}$. In other words, when $10 \mathrm{eV}$ have been deposited per atom, the atomic configuration is essentially different from before. The coincidence of this value with the energy of a few $\mathrm{eV}$ necessary for breaking a covalent bond is a strong argument in favor of the correctness of the proposed model but shows at the same time that the driving of dynamics by the beam is surprisingly efficient, as not all broken environments will relax towards a final state different from the initial state and also the mobile electrons can lose some of their energy directly to the phononic system as opposed to using it for breaking bonds. Thus, it seems that whenever a local rearrangement happens, it is not only that the handful of atoms involved in the broken and restored bonds are shifted by distances on the order of half of the typical atomic separations but that also a large number of atoms in the vicinity are relaxing by smaller distances due to the elastic coupling. This also explains why our IBM model, assuming infinitesimal steps, is so successful in explaining the $q$-dependent data.

We want to note that the proposed mechanism is different from direct knock-on damage, where the primary high-energy particle transfers its energy directly onto an atom. In our case, the maximum possible energy transfer would be about $2 \mathrm{eV}$ for the elastic collision of a $13 \mathrm{keV}$ photoelectron with a B or $\mathrm{O}$ atom, much less than the commonly quoted threshold of $25 \mathrm{eV}$ [36]. However, this latter value applies to the formation of a Frenkel pair in a crystal lattice, while for a network glass the threshold could be much lower. Here again it is the agreement with the data obtained by Pintori et al. [20], who used incident radiation of $8.1 \mathrm{keV}$, resulting in correspondingly smaller potential energy transfers, but observed dynamics comparable to our investigation, that allows us to rule out the dominance of this mechanism.

The picture of athermal beam-induced dynamics given above implies that the realized structure on the microscopic scale results essentially from the system following local minima in temporally varying potentials, thus leading to disturbances of the well-relaxed glassy state. We can now ask to what degree this purely athermal view is accurate. Indeed, the observation of deviations from a perfect linear relationship between dose rate and observed dynamics in $\left(\mathrm{Rb}_{2} \mathrm{O}\right)_{30}\left(\mathrm{~B}_{2} \mathrm{O}_{3}\right)_{70}$ as reported above can be seen as a hint in this direction: Possibly, the activation energy for rearrangements in the perturbed regions is lowered enough for thermal relaxations of the local structures to happen. The deviation from linearity at a dose rate of about $3 \mathrm{eV}$ per atom and second would then be the point where the rate of beam-induced changes surpasses the rate of thermal back relaxation in this most alkali-rich system, leading to a destabilization of the whole matrix and thus a superlinear acceleration of dynamics.

\section{CONCLUSIONS}

Studies of rubidium and cesium borate glasses with different ionic concentrations confirm the flux dependency of the atomic dynamics. The very high doses due to a hard $\mathrm{x}$-ray synchrotron beam affect the glass structure as probed by the diffraction curves by only a small amount, and no corresponding continuous acceleration of dynamics has been found. Hence, the beam induces only dynamical changes within the irradiated volume.

Further remarkable features are summarized as follows:

(i) For a given system, the relation between dose rate and dynamics is linear to good accuracy. Specifically, inherent thermal dynamics seem to be too slow to detect by aXPCS at room temperature.

(ii) Consistent with the rise of the glass transition temperature, the rate of dynamics at a given dose rate decrease with increasing alkali content. 
(iii) Comparing the decay rate $\Gamma$ and shape parameter $\beta$ of chemically equivalent $\mathrm{Rb}$ and $\mathrm{Cs}$ borate systems, it can be inferred that the beam-induced dynamics is faster for the alkali sites than for the borate network.

(iv) For all systems, the $q$-dependent behavior of the decay rate can be modeled satisfactorily by assuming that the atoms perform Brownian motion composed of infinitesimal jumps, subject to de Gennes narrowing.

Our studies serve to underline a point recently raised [17] and subsequently experimentally verified [18]: The absence of beam damage in the sense of a permanent modification of the sample's structure in a scattering experiment is a necessary, but not sufficient, condition for assuming the dynamical properties to be representative of their inherent values. Concerning experiments at next-generation ultralow emittance synchrotrons or free-electron laser sources using highly intense coherent beams that are based on femtosecond pulses, the beam-induced effect will become more and more relevant and therefore should be studied thoroughly to prevent misinterpretations of the experimental results. On the other hand, it is obvious that this possibility to directly study the dynamics of how hard x-ray photons below the knock-on energy threshold can rearrange matter on the atomic scale will lead to an improved understanding of the processes that do lead to permanent irradiation damage in materials and thus is of utmost technological relevance, for instance, for future fusion reactors.

\section{ACKNOWLEDGMENTS}

This work was funded by the Austrian Science Fund (FWF): Grant No. P28232-N36. We are grateful to Dr. M. Sprung (beamline scientist at DESY) for his help with performing the aXPCS experiments at beamline P10 at DESY.
[1] G. Vogl and B. Sepiol, in Diffusion in Condensed Matter: Methods, Materials, Models, 2nd ed., edited by P. Heitjans and J. Kärger (Springer, Berlin, 2005), pp. 65-91.

[2] M. Sutton, S. G. J. Mochrie, T. Greytak, S. E. Nagler, L. E. Berman, G. A. Held, and G. B. Stephenson, Nature (London) 352, 608 (1991).

[3] S. Brauer, G. B. Stephenson, M. Sutton, R. Brüning, E. Dufresne, S. G. J. Mochrie, G. Grübel, J. Als-Nielsen, and D. L. Abernathy, Phys. Rev. Lett. 74, 2010 (1995).

[4] A. Robert, J. Wagner, T. Autenrieth, W. Härtl, and G. Grübel, J. Chem. Phys. 122, 084701 (2005).

[5] G. Grübel, A. Madsen, and A. Robert, in Soft-Matter Characterization, edited by R. Borsali and R. Pecora (Springer, Dordrecht, 2008), pp. 953-995.

[6] L. Cipelletti and E. R. Weeks, in Dynamical Heterogeneities in Glasses, Colloids and Granular Media, edited by L. Berthier, G. Biroli, J.-P. Bouchaud, L. Cipelletti, and W. van Saarlos (Oxford University Press, Oxford, 2011), pp. 110-151.

[7] Y. Chushkin, C. Caronna, and A. Madsen, J. Appl. Crystallogr. 45, 807 (2012).

[8] S. O. Hruszkewycz, M. Sutton, P. H. Fuoss, B. Adams, S. Rosenkranz, K. F. Ludwig, W. Roseker, D. Fritz, M. Cammarata, D. Zhu, S. Lee, H. Lemke, C. Gutt, A. Robert, G. Grübel, and G. B. Stephenson, Phys. Rev. Lett. 109, 185502 (2012)

[9] H. Guo, G. Bourret, R. B. Lennox, M. Sutton, J. L. Harden, and R. L. Leheny, Phys. Rev. Lett. 109, 055901 (2012).

[10] M. Leitner, B. Sepiol, L.-M. Stadler, B. Pfau, and G. Vogl, Nat. Mater. 8, 717 (2009).

[11] M. Stana, M. Ross, and B. Sepiol, Diffus. Found. 2, 73 (2014).

[12] M. Ross, M. Stana, M. Leitner, and B. Sepiol, New J. Phys. 16, 093042 (2014).

[13] B. Ruta, Y. Chushkin, G. Monaco, L. Cipelletti, E. Pineda, P. Bruna, V. M. Giordano, and M. Gonzalez-Silveira, Phys. Rev. Lett. 109, 165701 (2012).
[14] V. M. Giordano and B. Ruta, Nat. Commun. 7, 10344 (2016).

[15] A. Fluerasu, A. Moussaïd, P. Falus, H. Gleyzolle, and A. Madsen, J. Synchrotron Radiat. 15, 378 (2008).

[16] J. Verwohlt, M. Reiser, L. Randolph, A. Matic, L. A. Medina, A. Madsen, M. Sprung, A. Zozulya, and C. Gutt, Phys. Rev. Lett. 120, 168001 (2018).

[17] M. Leitner, M. Stana, M. Ross, and B. Sepiol, Acceleration of atomic dynamics due to localized energy depositions under $\mathrm{X}$ ray irradiation, arXiv:1510.01918.

[18] B. Ruta, F. Zontone, Y. Chushkin, G. Baldi, G. Pintori, G. Monaco, B. Ruffle, and W. Kob, Sci. Rep. 7, 3962 (2017).

[19] A. C. Wright, Phys. Chem. Glasses: Eur. J. Glass Sci. Tech., Part B 51, 1 (2010).

[20] G. Pintori, G. Baldi, B. Ruta, and G. Monaco, Phys. Rev. B 99, 224206 (2019).

[21] J. E. Shelby, Introduction to Glass Science and Technology (Royal Society of Chemistry, Cambridge, 2005).

[22] M. Sutton, C. R. Phys. 9, 657 (2008).

[23] D. Abernathy, G. Grübel, S. Brauer, I. McNulty, G. Stephenson, S. Mochrie, A. Sandy, N. Mulders, and M. Sutton, J. Synchrotron Radiat. 5, 37 (1998).

[24] M. Leitner, Studying Atomic Dynamics with Coherent X-rays (Springer, Berlin, 2012).

[25] D. Lumma, L. B. Lurio, S. G. J. Mochrie, and M. Sutton, Rev. Sci. Instrum. 71, 3274 (2000).

[26] A. Madsen, R. L. Leheny, H. Guo, M. Sprung, and O. Czakkel, New J. Phys. 12, 055001 (2010).

[27] A. R. Sandy, L. B. Lurio, S. G. J. Mochrie, A. Malik, G. B. Stephenson, J. F. Pelletier, and M. Sutton, J. Synchrotron Radiat. 6, 1174 (1999).

[28] F. Berkemeier, S. Voss, Á. W. Imre, and H. Mehrer, J. NonCryst. Solids 351, 3816 (2005).

[29] A. H. Verhoef and H. W. den Hartog, J. Non-Cryst. Solids 182, 235 (1995).

[30] K. Niss, C. Dalle-Ferrier, G. Tarjus, and C. Alba-Simionesco, J. Phys.: Condens. Matter 19, 076102 (2007). 
[31] P. N. Segrè, O. P. Behrend, and P. N. Pusey, Phys. Rev. E 52, 5070 (1995).

[32] P. de Gennes, Physica (Amsterdam) 25, 825 (1959).

[33] M. Leitner and G. Vogl, J. Phys.: Condens. Matter 23, 254206 (2011).
[34] C. T. Chudley and R. J. Elliott, Proc. Phys. Soc. London 77, 353 (1961).

[35] H. Mehrer, Diffusion in Solids: Fundamentals, Methods, Materials, Diffusion-Controlled Processes (Springer, Berlin, 2007).

[36] E. A. Kenik and T. E. Mitchell, Philos. Mag. 32, 815 (1975). 